\title{
Lung atelectasis secondary to massive esophageal dilation in a patient with scleroderma
}

\author{
Charles St-Arnaud MD, Nicole Bouchard MD, Luc Lanthier MD MSc
}

\begin{abstract}
C St-Arnaud, N Bouchard, L Lanthier. Lung atelectasis secondary to massive esophageal dilation in a patient with scleroderma. Can Respir J 2010;17(4):e96-e98.

Esophageal dilation is a rare complication of scleroderma and CREST syndrome. A case of atelectasis secondary to right inferior bronchus compression by a massively dilated esophagus is described. The authors are unaware of any previous cases of atelectasis secondary to esophageal dilation in scleroderma.
\end{abstract}

Key Words: Atelectasis; CREST; Gastroenterology; Respiratory Medicine; Scleroderma

E sophageal dilation is a rare complication of scleroderma and CREST (calcinosis, Raynaud's syndrome, esophageal dysmotility, sclerodactyly, telangiectasia) syndrome. We report a case of atelectasis secondary to right inferior bronchus compression by a massively dilated esophagus. We are unaware of any previous cases of atelectasis secondary to esophageal dilation in scleroderma.

\section{CASE PRESENTATION}

A 69-year-old man presented to the emergency room with a three-week history of right pleuritic chest pain and progressive shortness of breath. His medical history was significant for scleroderma since he was 25 years of age, coronary artery disease and severe peripheral vascular disease. Because of major gastroesophageal reflux disease (GERD), he underwent an open transabdominal Nissen fundoplication and a hiatal hernia repair at 44 years of age. His GERD, which had dramatically improved following the surgery, had recurred since the age of 62 years.

On arrival to the emergency room, a chest $\mathrm{x}$-ray showed a massively dilated esophagus with right middle and lower pulmonary lobe atelectasis (Figure 1). A bronchoscopy revealed a crushed trachea, a severely narrowed right intermediate bronchus and a small amount of purulent secretions. There was no endoluminal tumour and cytology specimens were negative. A culture of bronchial secretions sampled during bronchoscopy was negative. A chest computerized tomography (CT) scan showed a dilated esophagus full of food debris, with complete right lower lobe and partial right-middle lobe atelectasis (Figure 2).

Because of the high risk of aspiration associated with esophagoscopy, a barium swallow was performed and confirmed the severe esophageal dilation with gastroesophageal junction stenosis (Figure 3). No peristalsis was observed in the distal esophagus. Although the examination was limited due to the

\section{Atélectasie pulmonaire secondaire à une dilatation œsophagienne massive chez un patient atteint de sclérodermie}

La dilatation œsophagienne est une rare complication de la sclérodermie et du syndrome CREST. On décrit ici un cas d'atélectasie secondaire à une compression de la bronche inférieure droite par un œsophage massivement dilaté. Les auteurs ne connaissent aucun autre cas d'atélectasie secondaire à une dilatation œesophagienne dans la sclérodermie.

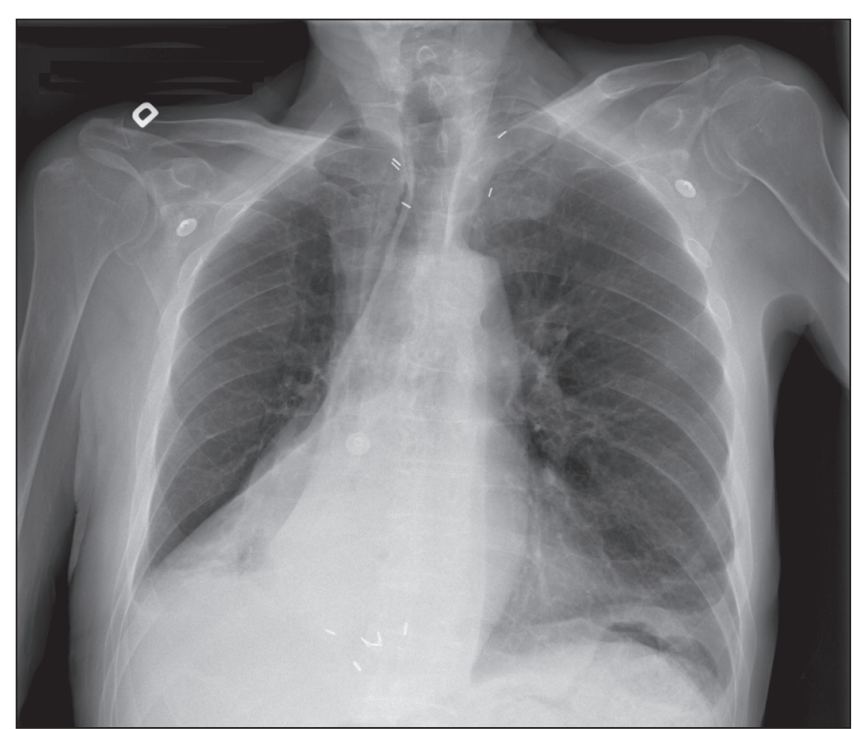

Figure 1) Anterior chest $x$-ray. Complete right lower lobe and partial right middle lobe atelectasis

presence of food debris, no mucosal ulceration or cancer was suspected. The patient declined an esophagoscopy in favour of possible therapeutic distal esophageal dilation.

The patient's dyspnea and shortness of breath improved with a seven-day course of moxifloxacin. Given the patient's severe comorbidities from his peripheral vascular disease, a conservative approach to treatment was decided. He was discharged with a proton pump inhibitor, and was advised to keep the head of his bed at a $30^{\circ}$ angle and to eat in the sitting position.

\section{DISCUSSION}

Esophageal involvement in scleroderma is common and affects up to $90 \%$ of patients, most of whom are asymptomatic $(1,2)$.

Department of Medicine, Université de Sherbrooke, Sherbrooke, Quebec

Correspondence: Dr Charles St-Arnaud, Department of Medicine, Faculty of Medicine, Université de Sherbrooke, 3001, 12ème Avenue Nord,

Sherbrooke, Quebec J1H 5N4. E-mail charles.st-arnaud@usherbrooke.ca 

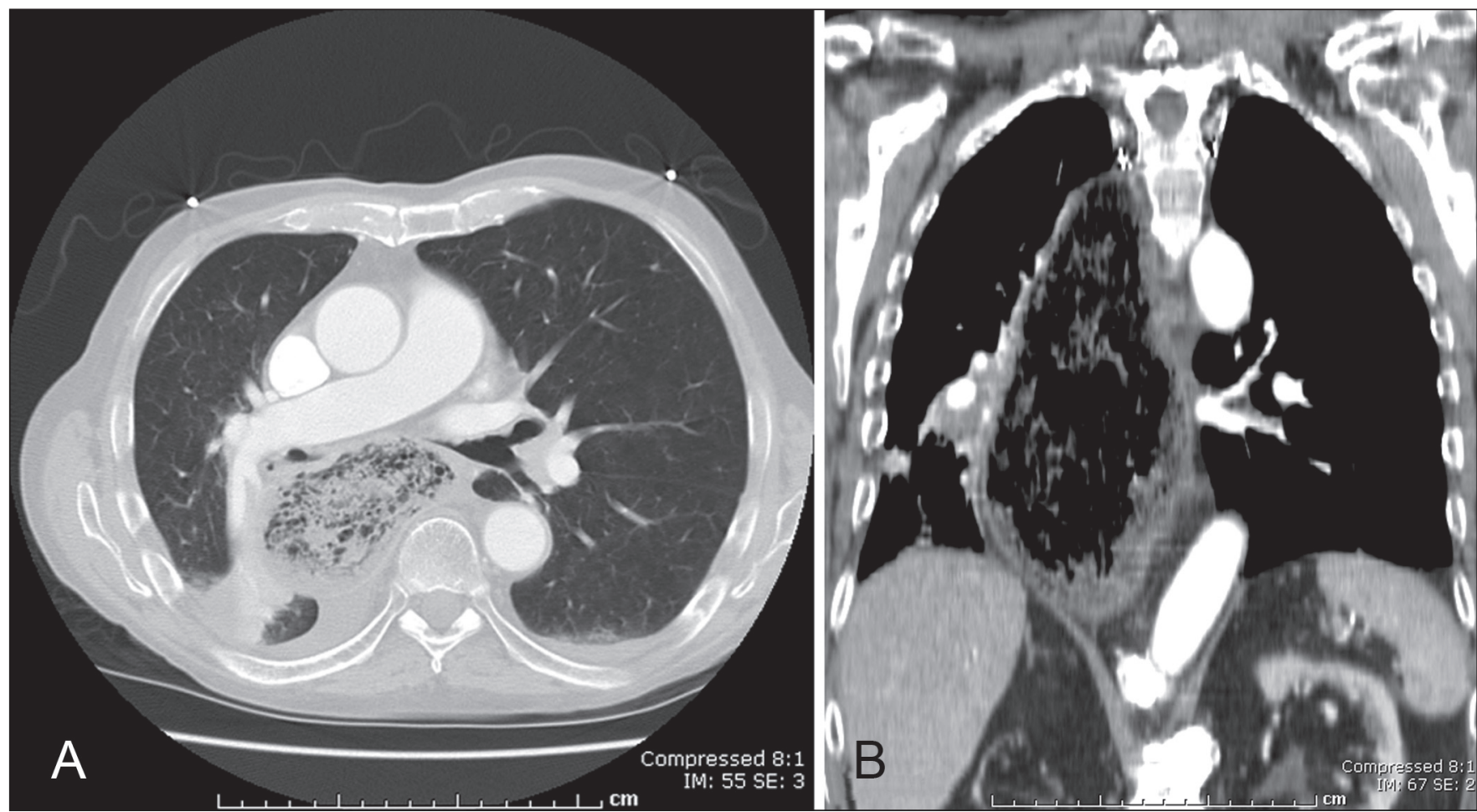

Figure 2) Chest computed tomography scan with axial (A) and coronal (B) views. Severe esophageal dilation is visible with right lower lobe atelectasis and right main bronchus compression

The distal two-thirds of the esophagus is usually involved and is associated with a decrease in lower esophageal sphincter tone and poor esophageal motility. Decreased lower esophageal sphincter tone permits the passage of gastric contents into the esophagus where impaired peristalsis leads to prolonged esophageal exposure to the refluxate (2). In time, esophagitis with stricture formation can develop.

Esophageal surgery is indicated for patients with documented GERD and with persistent symptoms necessitating medical treatment (3). GERD complications despite medical treatment, such as esophageal erosions, esophagitis, stricture formation or recurrent aspirations, are also surgical indications $(3,4)$. Since the introduction of proton pump inhibitors in the 1990s, medical failure has become less common and esophageal surgery is performed mostly in patients with poor adherence to medication.

Among the complications related to surgery, there is recurrence of reflux, esophageal obstruction and hiatus hernia. No case of significant esophageal dilation following Nissen fundoplication has been reported. In patients with scleroderma, esophageal aperistalsis increases the risk of complication. Although only small case series of postesophageal surgery in patients with scleroderma are available, the rate of postoperative reflux recurrence and dysphagia can be as high as $71 \%$ (5). New surgical techniques have shown encouraging results in patients with esophageal dysmotility (5). Nevertheless, few studies have had a follow-up period of longer than six years $(6,7)$.

Pulmonary complications in scleroderma patients, such as aspiration pneumonitis, pulmonary hypertension and interstitial lung disease, have been reported in up to $70 \%$ of patients. However, lung atelectasis secondary to esophageal compression of the main or intermediate bronchus is extremely rare. A Medline search, including data from 1950 up to April 2009,

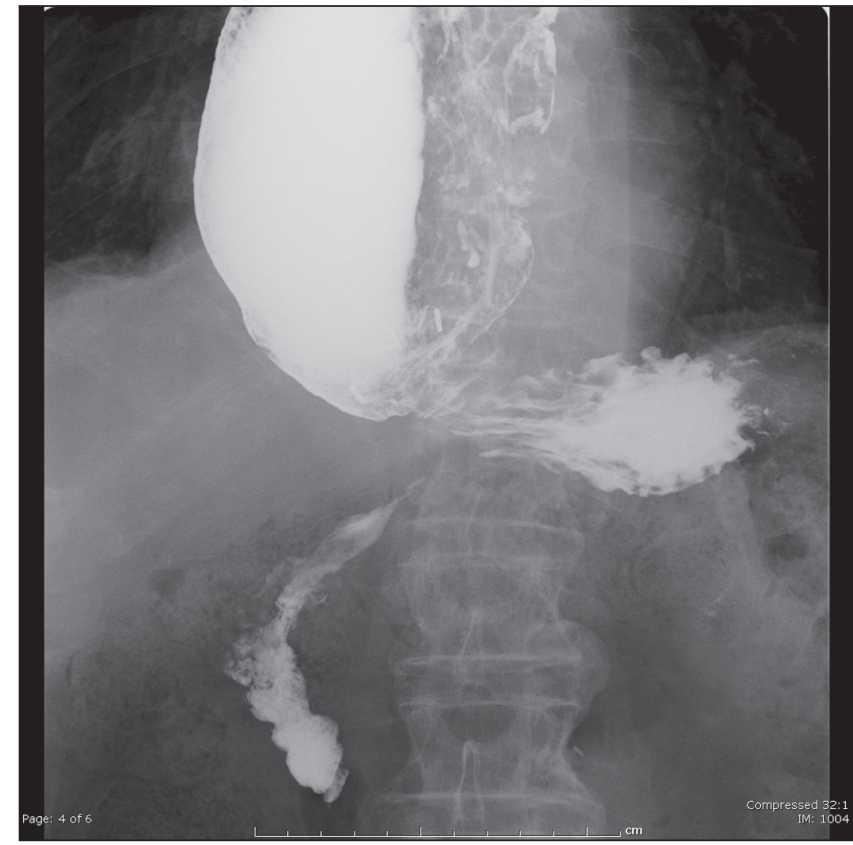

Figure 3) Barium swallow showing a dilated esophagus with gastroesophageal junction stenosis. The esophageal diameter is greater than $10 \mathrm{~cm}$

was performed. We found one report (8) of right lung atelectasis in a patient with severe esophageal dilation secondary to achalasia. Left lung atelectasis secondary to traumatic esophageal hematoma has also been reported (9).

In our patient, we believe that postoperative gastroesophageal stenosis developed, which, combined with deteriorating esophageal peristalsis, led to massive esophageal dilation with 
compression of the right lower and middle lobe bronchus. Our patient may have developed bronchial infection from food aspiration; however, the symptoms evolving over a three-week period, the small amount of secretion seen during the bronchoscopy, as well as a negative culture suggest that extrinsic esophageal compression was the main cause of his atelectasis. We believe that the present case is the first report of atelectasis secondary to esophageal dilation in a patient with scleroderma.

\section{CONCLUSION}

Esophageal involvement in scleroderma is a frequent complication. Although most patients improve with medical therapy, some remain extremely symptomatic and surgery may be the only therapeutic option. Patients and physicians must be aware

\section{REFERENCES}

1. Systemic sclerosis and scleroderma variants: Clinical aspects. In: Koopman WJ. Moreland LW, eds. Arthritis and Allied Conditions: A Textbook of Rheumatology, 15th edn. Philadelphia: Lippincott Williams \& Wilkins 2005:1633-80.

2. Young MA, Rose S, Reynolds JC. Gastrointestinal manifestations of scleroderma. Rheum Dis Clin North Am 1996;22:797-823.

3. Kahrilas PJ, Shaheen NJ, Vaezi MF, et al. American Gastroenterological Association medical position statement on the management of gastroesophageal reflux disease. Gastroenterology 2008;135:1383-91.

4. Surgical therapies for gastroesophageal reflux disease. In: Shields TW, LoCicero J, Ponn RB, Rusch VW, eds. General Thoracic Surgery, 6th edn. Philadelphia: Lippincott Williams \& Wilkins, 2005:2173-85. of the high recurrence rate and complications after surgery. Severe esophageal dilation following surgery can be a very late and under-recognized complication in scleroderma patients with esophageal dysmotility. However, atelectasis secondary to massive esophageal dilation remains a very unusual complication.

AUTHOR CONTRIBUTIONS: All authors declare that they participated in the writing of the manuscript and have seen and approved the final version.

CONFLICTS OF INTEREST: The authors have no commercial or other associations that might pose a conflict of interest in connection with the present article.

5. Kent MS, Luketich JD, Irshad K, et al. Comparison of surgical approaches to recalcitrant gastroesophageal reflux in the patient with sclerodema. Ann Thorac Surg 2007;84:1710-6.

6. Watson DI, Jamieson GG, Bessell JR, Devitt PG. Laparoscopic fundoplication in patients with an aperistaltic esophagus and gastroesophageal reflux. Dis Esophag 2006;19:94-8.

7. Poirier NC, Taillefer R, Topart P, Duranceau A. Antireflux operations in patients with scleroderma. Ann Thorac Surg 1994;58:66-73.

8. Liang CY. Lin MS. Images in clinical medicine: Achalasia. N Engl J Med 2009;360:801.

9. Rigal BR, Leteurnier JC, Lebert C, Blanloeil Y. Post-traumatic hematoma of the esophagus. Ann Fr Anesth Reanim 1998;17:1160-3. 


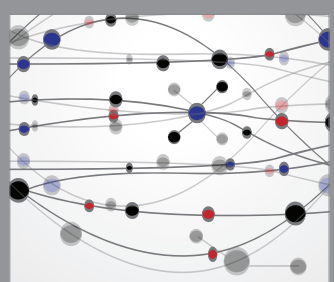

The Scientific World Journal
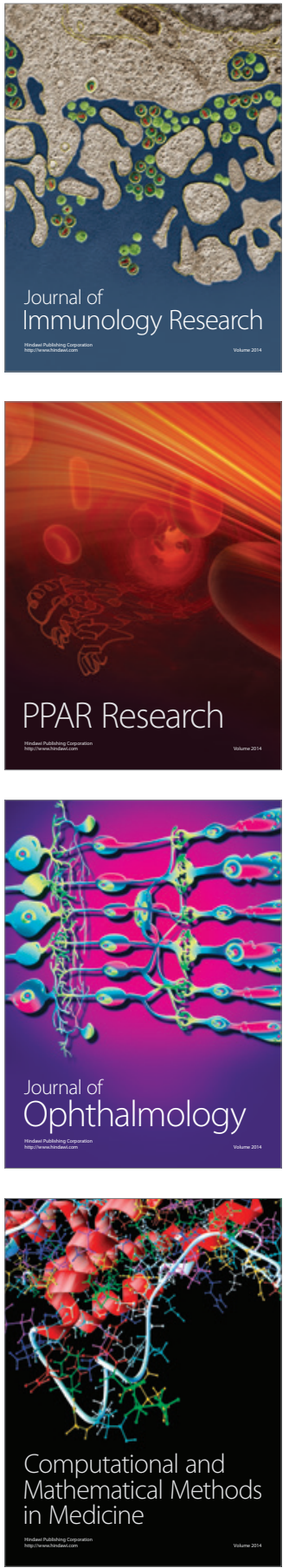

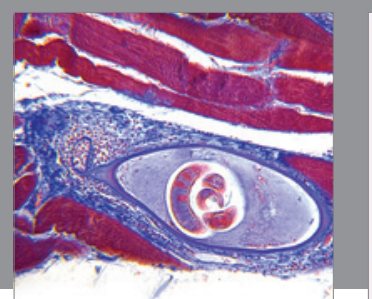

Gastroenterology Research and Practice

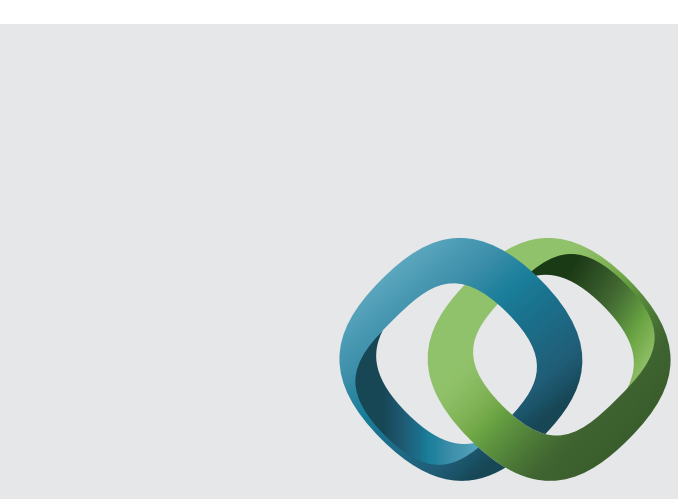

\section{Hindawi}

Submit your manuscripts at

http://www.hindawi.com
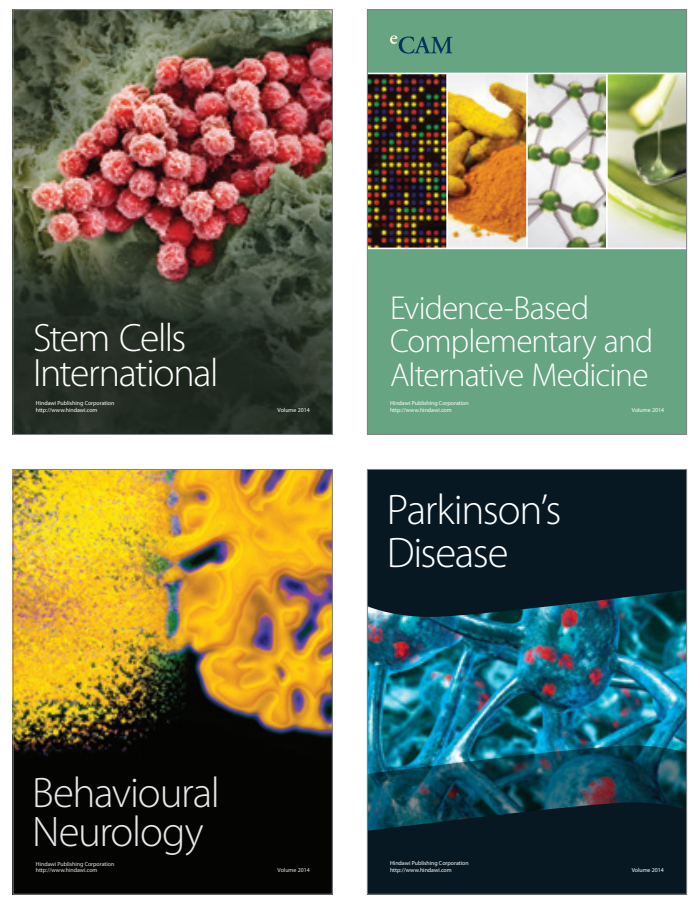
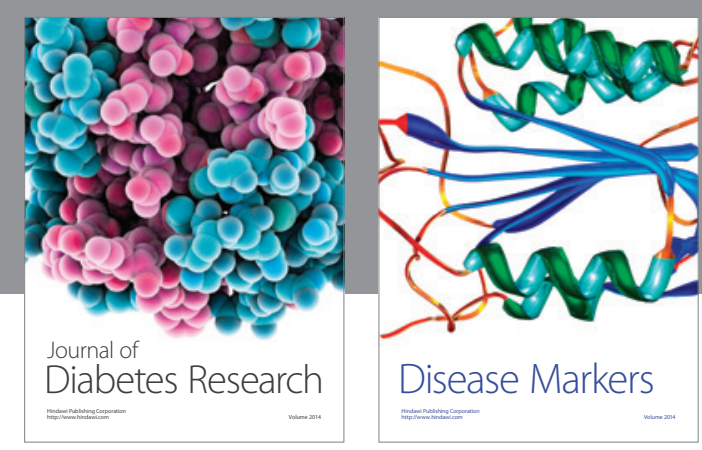

Disease Markers
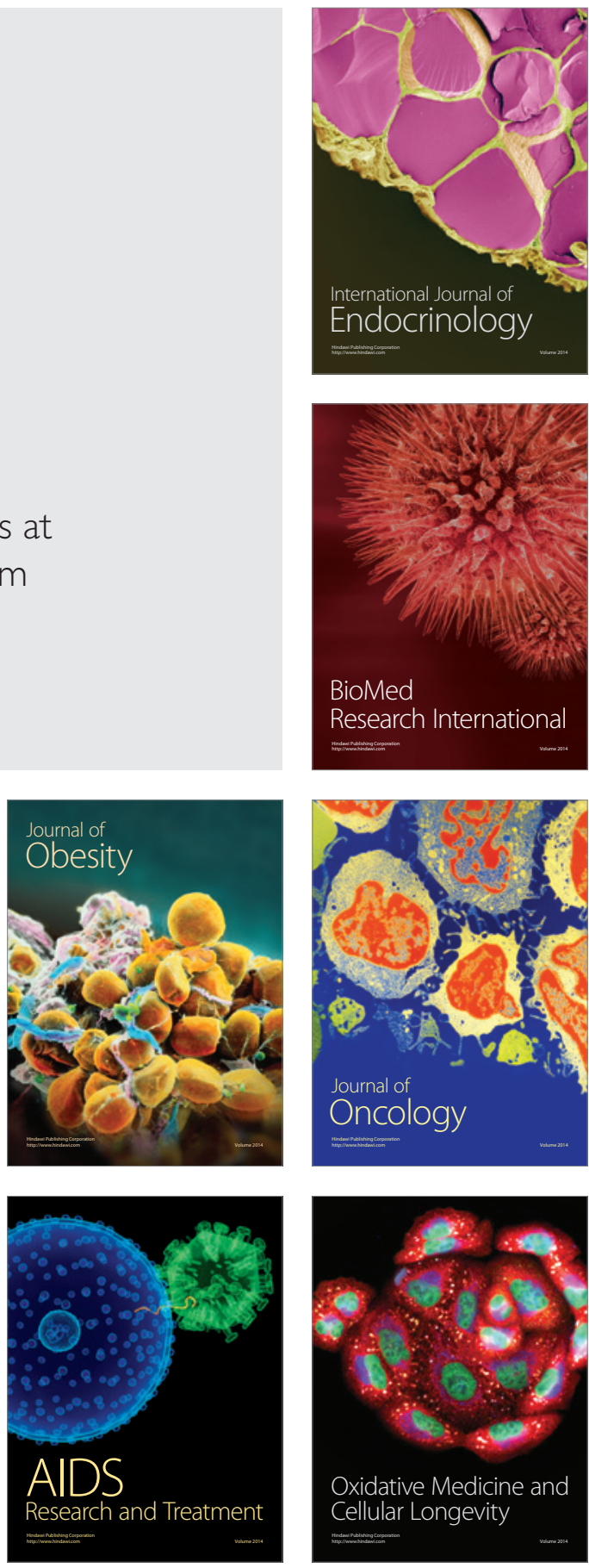